\title{
Antiretroviral adherence interventions: what works and what does not work
}

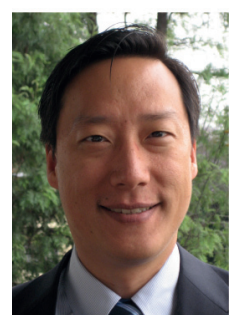

\section{"Understanding what antiretroviral adherence interventions work is ... vitally important in preventing treatment failure and spread of antiretroviral resistance."}

Michael H Chung

Departments of Global Health, Medicine \& Epidemiology, University of Washington, 325 Ninth Ave, Box 359909, Seattle, WA 98104, USA = Tel.: +1 2065434278 = Fax: +1 2065434818 nnchung@u.washington.edu

Since 1997, the introduction of combined antiretroviral therapy (cART) has revolutionized the care of individuals infected with HIV and has significantly extended lifespans to nearly normal levels $[1,2]$. However, viral resistance and therapeutic failure can develop when patients do not adhere to their antiretroviral (ARV) medications. Understanding what antiretroviral adherence interventions work is therefore vitally important in preventing treatment failure and the spread of ARV resistance.

To answer the seemingly simple question of what adherence interventions work, it must first be understood what it means for an intervention to successfully 'work'. This is more challenging than it appears because of the difficulty in defining and measuring good adherence.

Defining the level of adherence that is required to consistently and adequately suppress plasma HIV-1 RNA differs according to the composition of cART. Maintaining adherence above $95 \%$ was originally considered necessary to avoid virologic failure [3]. For a one-pill fixed dose combination that is taken twice a day, this requires a patient not to miss more than a day and a half of drugs or less than three pills each month. However, this restriction now appears to apply primarily to ARV regimens that are based on unboosted protease inhibitors, such as indinavir or saquinavir, which have a short half-life in vivo [4]. Regimens that are based on non-nucleoside reverse transcriptase inhibitors, such as nevirapine or efavirenz, require less stringent adherence and are adequate at adherence levels down to $80 \%$.

There are several ways to measure adherence and each method can give different results. These methods include directly observing a patient while they are taking their pills, measuring drug levels from body compartments such as blood, urine or hair, counting the number of pills the patient has taken, using pharmacy refill records, and capturing self-reported adherence [5]. While directly observing a patient or measuring biological drug levels is more accurate, it is time-consuming and expensive to administer. Relying on patients to report how adherent they have been is subject to bias, but is the easiest way to measure adherence and is therefore the most common method used in adherence studies. The challenges of defining and measuring good adherence exacerbate the problem of a relative paucity of randomized controlled trials on ARV adherence [6].

\section{What adherence interventions work?}

Despite these caveats, it is possible to identify several adherence interventions that work. These interventions can be broken down into the following categories: simplifying cART; decreasing structural barriers to care; applying cognitive and behavioral therapies; and strengthening social support networks.

\section{Simplifying ARV regimens}

Drug regimens that are easier to take are easier to adhere to [7]. This includes limiting the size, number, side effects and frequency of pills taken per day [8]. Most patients find taking one small pill once a day with minimal side effects is easier to adhere to than a regimen that is burdened with numerous side effects and requires three large pills to be taken three times a day. Consolidated drug regimens are also less likely to draw attention to drug use that may be stigmatizing to a patient trying to conceal her HIV status [9]. Simplifying cART is one of the easiest ways to increase adherence and should be the first step in adherence promotion.
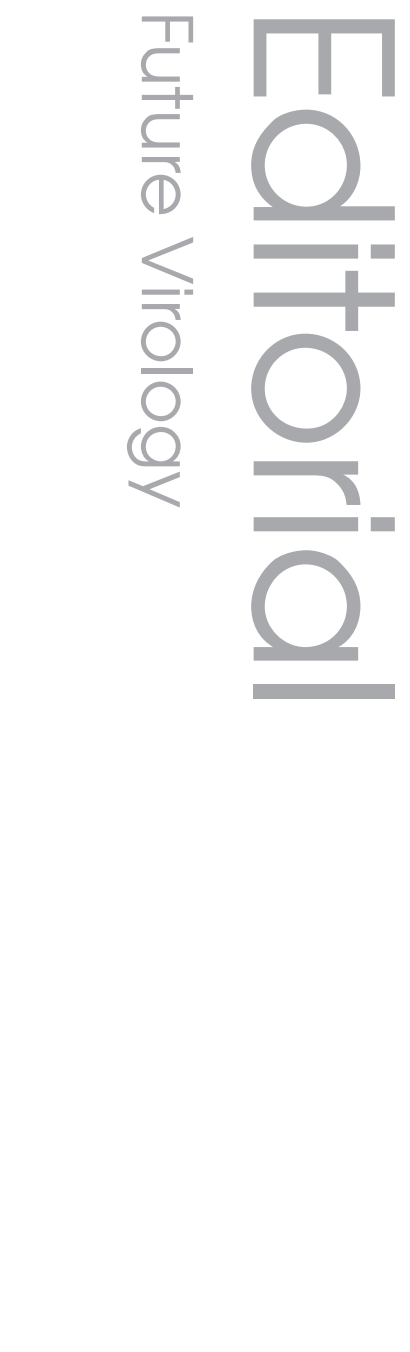

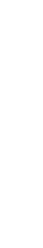




\section{Surmounting structural barriers}

Interventions that reduce the time, effort, and money required by patients to access their ARVs improve adherence [10]. This can be done in a number of ways. One of the most straightforward is to support the health care facilities that provide ARVs. Pharmacies or clinics that are adequately staffed and consistently stocked with $A R V$ s reduce patient waiting times and the chances that the drugs will be out of supply. Adherence increases if ARVs are free and a patient does not have to expend resources traveling to clinic, storing drugs safely, or buying food to take with the medications. This can be addressed by bringing drugs to the home (e.g., directly administered therapy), giving fare for transportation, providing shelter and improving food security [11]. Improving access to drugs is an essential means of improving drug adherence and cannot be taken for granted, especially in resource-limited settings and among indigent patients [12].

\section{Implementing cognitive \\ \& behavioral approaches}

There are multiple psychological and cognitive interventions that are associated with improved adherence [13]. These methods include decreasing drug and alcohol addictions, empowering the patient with self-management skills, motivating constructive behavior, treating depression, educating the patient and providing emotional support [14]. These interventions are typically performed during face-to-face interactions and involve counseling, learning and problem-solving. Direct human contact is increasingly being replaced by novel technological methods such as pagers, videoconferencing through the internet and text-messaging cell phones which are easier to implement, cheaper to scale-up and similarly successful in improving ARV adherence [15]. These interventions are effective, in part, because they support the patient's perception that the treatment clinic is committed to their well-being.

\section{Strengthening social support}

Ensconcing a patient within a supportive social network improves adherence to ARVs. Children particularly rely on responsible caregivers who will help them take their medications on time. Family, friend and spousal support is associated with better ARV adherence among adults as well. Given the stigma that is associated with HIV infection, disclosing one's HIV status is an important step in acknowledging the challenges of living with the disease and obtaining support [16]. Implementing a buddy system where a friend or family member is identified by patients to help them maintain adherence can deal with some of these issues [17]. Social networks not only support the patient but generate obligations that can have positive effects on adherence. Adhering to ARV drugs in order to stay alive and earn money and/or care for dependent family members is a powerful motivating force that may explain the higher rates of adherence found in resource-limited settings [18].

\section{What does not work?}

The challenge is that while the above interventions are found to be helpful, none are guaranteed to be effective in every situation. ARV adherence is dictated by patient choice and individual circumstances that cannot be completely controlled. Interventions that fail, then, are those that do not adapt to conditions that impact individual adherence and personal motivations. Relying on devices such as alarms or pill boxes with the intention to 'fix' adherence by reminding patients when to take their medications is not sufficient to improve adherence [14]. These items can assist patients who have decided to adhere to their ARV regimen or among those with memory problems, but lack efficacy without motivation or need.

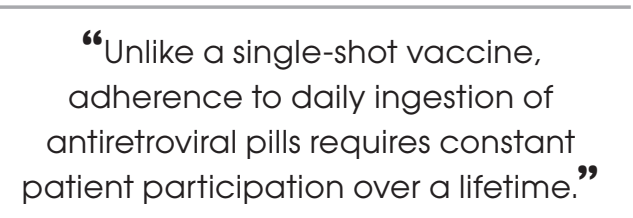

The impact of adherence interventions varies between groups and individuals. Adherence results can differ according to gender, sexual preference, socioeconomic status, age, psychological state, baseline adherence, drug dependency and illness. Poor adherence issues among HIV-positive injecting drug users in urban America differ from HIV-positive noninjecting drug users living in rural sub-Saharan Africa. Illicit drug use among the former responds to concomitant methadone treatment as an adherence intervention [19]. However, food insecurity and poor healthcare access among the latter responds better to subsidization of transportation costs and food supplementation [11]. As ARV therapy is extended to HIVnegative individuals to prevent the acquisition of HIV, it is increasingly important to identify and understand the personal relevance of adherence [20]. 
In summary, defining adherence interventions that work is challenged by varying methodologies and a relative lack of rigorously determined results. Nevertheless, there are several good interventions that improve ART adherence among HIV-positive individuals. Prescribing cART that is simpler to take and easier to access is a key first step. Some cognitive and behavioral interventions help, as well as a strong social support network. Yet, none of these interventions is a panacea. Unlike a single-shot vaccine, adherence to daily ingestion of ART pills requires constant patient participation over a lifetime. Understanding the patient and their circumstances is therefore essential to any successful adherence intervention. In this way, caring for the whole patient and not just their HIV disease is the best adherence intervention.

\section{Financial \& competing interests disclosure \\ The author has no relevant affiliations or financial involvement with any organization or entity with a financial interest in or financial conflict with the sub- ject matter or materials discussed in the manuscript. This includes employment, consultancies, honoraria, stock ownership or options, expert testimony, grants or patents received or pending, or royalties. \\ No writing assistance was utilized in the production of this manuscript.}

\section{Bibliography}

1. Antiretroviral Therapy Cohort Collaboration. Life expectancy of individuals on combination antiretroviral therapy in high-income countries: a collaborative analysis of 14 cohort studies. Lancet 372(9635), 293-299 (2008).

2. Mills EJ, Bakanda C, Birungi J et al. Life expectancy of persons receiving combination antiretroviral therapy in low-income countries: a cohort analysis from Uganda. Ann. Intern. Med. 155(4), 209-216 (2011).

3. Paterson DL, Swindells S, Mohr J et al. Adherence to protease inhibitor therapy and outcomes in patients with HIV infection. Ann. Intern. Med. 133(1), 21-30 (2000).

4. Bangsberg DR. Less than $95 \%$ adherence to nonnucleoside reverse-transcriptase inhibitor therapy can lead to viral suppression. Clin. Infect. Dis. 43(7), 939-941 (2006).

5. Berg KM, Arnsten JH. Practical and conceptual challenges in measuring antiretroviral adherence. J. Acquir. Immune. Defic. Syndr. 43(Suppl. 1), S79-S87 (2006).

6. De Bruin M, Viechtbauer W, Schaalma HP, Kok G, Abraham C, Hospers HJ. Standard care impact on effects of highly active antiretroviral therapy adherence interventions: a meta-analysis of randomized controlled trials. Arch. Intern. Med. 170(3), 240-250 (2010).

7. Nachega JB, Mugavero MJ, Zeier M, Vitória M, Gallant JE. Treatment simplification in HIV-infected adults as a strategy to prevent toxicity, improve adherence, quality of life and decrease healthcare costs. Patient Prefer. Adherence 5(1), 357-367 (2011).
8. Parienti JJ, Bangsberg DR, Verdon R, Gardner EM. Better adherence with once-daily antiretroviral regimens: a metaanalysis. Clin. Infect. Dis. 48(4), 484-488 (2009).

9. Van Tam V, Pharris A, Thorson A, Alfven T, Larsson M. "It is not that I forget, it's just that I don't want other people to know": barriers to and strategies for adherence to antiretroviral therapy among HIV patients in Northern Vietnam. AIDS Care 23(2), 139-145 (2011).

10. Hardon AP, Akurut D, Comoro C et al. Hunger, waiting time and transport costs: time to confront challenges to ART adherence in Africa. AIDS Care 19(5), 658-665 (2007).

11. Tuller DM, Bangsberg DR, Senkungu J, Ware NC, Emenyonu N, Weiser SD. Transportation costs impede sustained adherence and access to HAART in a clinic population in southwestern Uganda: a qualitative study. AIDS Behav. 14(4), 778-784 (2010).

12. Pyne-Mercier LD, John-Stewart GC, Richardson BA et al. The consequences of post-election violence on antiretroviral HIV therapy in Kenya. AIDS Care 23(5), 562-568 (2011).

13. Rueda S, Park-Wyllie LY, Bayoumi AM et al. Patient support and education for promoting adherence to highly active antiretroviral therapy for HIV/AIDS. Cochrane. Database Syst. Rev. 3, CD001442 (2006).

14. Chung MH, Richardson BA, Tapia K et al. A randomized controlled trial comparing the effects of counseling and alarm device on HAART adherence and virologic outcomes. PLoS Med. 8(3), E1000422 (2011).
15. Lester RT, Ritvo P, Mills EJ et al. Effects of a mobile phone short message service on antiretroviral treatment adherence in Kenya (WelTel Kenya1): a randomised trial. Lancet 376(9755), 1838-1845 (2010).

16. Stirratt MJ, Remien RH, Smith A, Copeland OQ, Dolezal C, Krieger D. The role of HIV serostatus disclosure in antiretroviral medication adherence. AIDS Behav. 10(5), 483-493 (2006).

17. Kunutsor S, Walley J, Katabira E et al. Improving clinic attendance and adherence to antiretroviral therapy through a treatment supporter intervention in Uganda: a randomized controlled trial. AIDS Behav. doi:10.1007/s10461-011-9927-9 (2011) (Epub ahead of print).

18. Ware NC, Idoko J, Kaaya $S$ et al. Explaining adherence success in sub-Saharan Africa: an ethnographic study. PLoS Med. 6(1), E11 (2009).

19. Roux P, Carrieri MP, Villes V et al. The impact of methadone or buprenorphine treatment and ongoing injection on highly active antiretroviral therapy (HAART) adherence: evidence from the MANIF2000 cohort study. Addiction 103(11), 1828-1836 (2008).

20. Baeten JM. Antiretroviral pre-exposure prophylaxis for HIV-1 prevention among heterosexual African men and women: the Partners PrEP study. HIV-1-infected adults. Presented at: 6th IAS Conference on HIV Pathogenesis, Treatment and Prevention. Rome, Italy, 17-20 July 2011 (Abstract MOAX0106). 